\title{
Forecasting future transport
}

J. I. Gershuny, of the Science Policy Research Unit, University of Sussex, discusses the recommendations of the Department of Transport report of the Advisory Committee on Trunk Road Assessment (HMSO, January 1978)

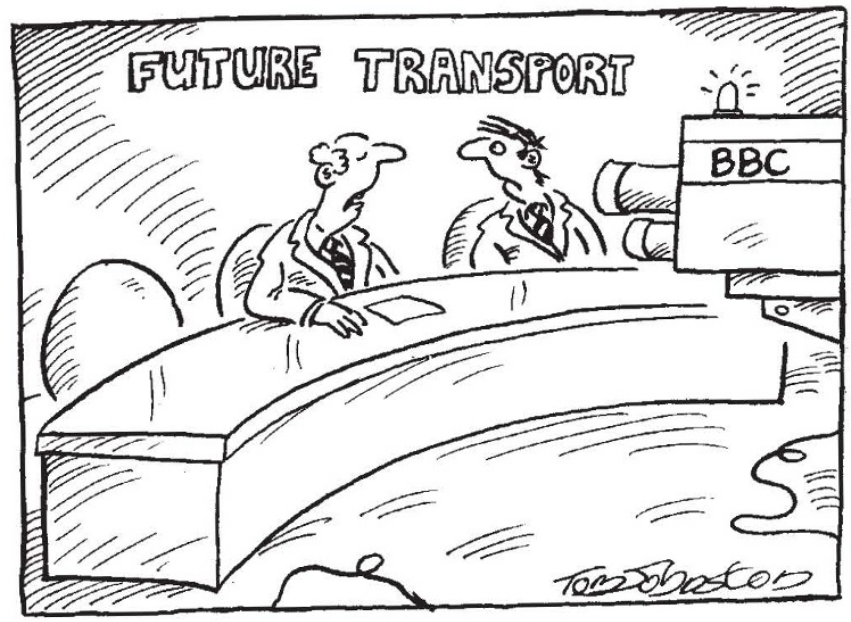

"We were due to have a spokesman from $T R R L$ here tonight .. . but he's been held up in the traffic!"

OFFICIAL procedure in the UK for assessing trunk road construction schemes has, in recent years, been the subject of increasing criticism. Statisticians and transport experts have claimed that the procedures are technically faulty. Local objectors to road building schemes complain that the obscurity of the methods prevents their participation in the planning process. The Leitch Committee, which reported recently, supported the technical criticisms, but did not suggest much practical help for protesters.

Decisions to build trunk roads in the UK are based on two technical procedures. One is a forecasting exercise carried out by a group at the Transport and Road Research Laboratory (TRRL) in Buckinghamshire. The other is a computerised cost benefit analysis package called COBA, developed by the UK Department of Transport and its predecessors. Both have come under increasing criticism.

The TRRL forecasting procedure is described in the report LR650 published by the laboratory. This document makes forecasts of vehicle numbers extending into the quite distant future - the final time horizon is the year 2010. It puts forward three alternative estimates of the car population of the UK at this date-25.0, 25.9 and 26.2 million cars, or $0.43,0.44$ and 0.45 cars per head of population respectively (LR650, p15). These alternative estimates are very close together by the normal standards of social forecasting; can we really be this certain?

The reason for the small range of the alternative forecasts is to be found in the forecasting procedure adopted by the TRRL group. The predictions are based on the assumption that the pattern of growth is logistic (Sshaped) over time. A logistic growth model tends towards a given saturation level at a rate determined by some causal variable or variables-in the TRRL case, national income, motoring costs and time. So the high estimate of 26.2 million cars in 2010 involves an assumption of high national income growth and low growth in motoring costs, the low 25.0 estimate assumes low income and high costs growth, and the middle one involves medium costs and growth.

A first reason that these estimates should bunch so closely together is that the very steady historical pattern of growth of vehicle numbers in the fifteen years preceding the fore- cast showed little fluctuation with variations in incomes and costs. So when calibrating the model, the future effect of variations of prices and incomes is similarly assumed to be small in relation to the effect of the passage of time. Even large assumed fluctuations make little difference to the forecast-because on past evidence the price and income elasticities of demand for private cars is low. Sceptics doubt that historically derived elasticities necessarily hold for the future.

A second reason for the bunching is that built into the structure of the model is a tendency for the effect of the variables on the forecast to be progressively suppressed over time as the value of the saturation asymptote is approached. The saturation level assumed in LR650 is 0.45 cars per head, and since the final predictions are so very close to this we might reasonably assume that the causal variables are contributing very little to the final result. Finally, the saturation level itself is very much a matter of speculation. In spite of sophisticated presentation, the results of the exercise come down to little more than guesses.

The cost-benefit assessment package, COBA, is rather more straight-forward, being, of course, subject to all the standard criticisms of cost-benefit analysis. The system requires that planners put money values on monuments and artifacts that have no natural economic worth, but nevertheless have considerable cultural value. Furthermore, cost-benefit analyses inevitably leave some costs completely unconsidered; COBA itself takes virtually no account of environmental damage caused by road building schemes. And even were all conceivable impacts taken into account, the effect of using a market-based system of valuation is that the preferences of the rich are given much more weight than those of the poor in the assessment.

Further COBA's assessment of future benefits is based in part on the predictions of future car numbers-giving rise to the suspicion that the TRRL vehicle forecasts may be self-fulfilling. However arbitrary the original predictions are, if, on the basis of these forecasts, new roads are built, and if, as many people suspect, the number of cars increases to fill the available road space, then the volume of traffic may well grow to the forecast level-whatever that level is! Hardly a rational way of formulating policy.

It was this sort of criticism that led to the appointment of the committee of enquiry into trunk road assessment under the chairmanship of Sir George Leitch. The committee's report contains an extremely thorough and lucid review of the assessment methods as currently practised. It examines vehicle forecasting procedures and methods of economic and environmental evaluation and compares them with practices in France, the USA and West Germany.

The Leitch report recommends that the current vehicle forecasting methods should be scrapped. To replace them the committee suggests a modelling procedure that gives much more weight to those causal factors-such as household organisation, geographical location of facilities and the availability of public transport-which actually determine people's desires to own and use cars. Such models would in particular be sensitive to the effects of public policies altering the balance of costs and convenience between public and private transport; some observers suggest that encouraging the use of public transport relative to private might in the long term have an important effect on the growth of car numbers, but the present forecasting procedure is quite insensitive to such changes in policy. This sort of conditional information about the effects on vehicle use of alternative feasible public policies would be much more useful to policymakers than the present unconditional forecasts. The report-as far as it goes-is to be welcomed. 